\title{
Mini International Neuropsychiatric Interview (MINI): validação de entrevista breve para diagnóstico de transtornos mentais

\author{
Mini International Neuropsychiatric Interview (MINI): validation of a \\ short structured diagnostic psychiatric interview
}

Patrícia Amorim*

Faculdade Pitié-Salpêtrière da Universidade de Paris VI, França e Núcleo de Atenção Psicossocial (NAPS) Novo Mundo, Goiânia

Resumo Objetivos: O MINI é uma entrevista diagnóstica padronizada breve (15-30 minutos), compatível com os critérios do DSM-III-R/IV e da CID-10, que é destinada à utilização na prática clínica e na pesquisa em atenção primária e em psiquiatria, e pode ser utilizada por clínicos após um treinamento rápido (de 1 a 3 horas). A versão Plus do MINI, mais detalhada, gera diagnósticos positivos dos principais transtornos psicóticos e do humor do DSM-IV. Este artigo apresenta os resultados de quatro estudos de validação do instrumento, realizados na Europa e nos EUA.

Métodos: Os estudos 1 (França) e 2 (EUA) testaram a confiabilidade - entre avaliadores e teste-reteste - da versão DSM-III-R do MINI ( $n=84$, sendo 42 pacientes psiquiátricos de cada centro) e sua validade com relação ao CIDI ( $n=346$, sendo 296 pacientes psiquiátricos e 50 controles) e ao SCID-P ( $n=370$, sendo 308 pacientes psiquiátricos e 62 controles), respectivamente. O estudo 3 testou a validade de diagnósticos gerados por clínicos gerais usando o MINI (DSM-IV) com relação aos diagnósticos clínicos habituais de psiquiatras, em 409 pacientes de centros de atenção primária de quatro países (França, Espanha, Itália e Reino Unido). O estudo 4 testou a confiabilidade entre avaliadores ( $\mathrm{n}=20$ pacientes psiquiátricos) e a validade dos módulos Transtornos Psicóticos, Depressão e Mania do MINI Plus - DSM IV (n=104 pacientes psiquiátricos) com relação a dois critérios de referência: diagnósticos do CIDI e diagnósticos clínicos de psiquiatras. Análises quantitativas (índices de concordância e de validade) e qualitativas (razões de discordância) foram realizadas.

Resultados: Os índices de confiabilidade do MINI (estudos 1 e 2) e do MINI Plus (estudo 4) foram globalmente satisfatórios. Comparados a vários critérios de referência (CIDI, SCID-P, opinião de peritos), em diferentes contextos (unidades psiquiátricas e centros de atenção primária), o MINI e o MINI Plus mostraram qualidades psicométricas similares às de outras entrevistas diagnósticas padronizadas mais complexas, permitindo uma redução de $50 \%$ ou mais no tempo da avaliação. Análises qualitativas identificaram dificuldades e erros diagnósticos ligados aos casos, métodos de avaliação e critérios de diagnósticos estudados. Modificações foram introduzidas para corrigir os problemas identificados e otimizar as propriedades psicométricas do MINI e do MINI Plus.

Conclusões: O MINI e sua versão Plus são adaptados ao contexto clínico e à avaliação de pacientes mais graves, e representam uma alternativa econômica para a seleção de pacientes, segundo critérios internacionais, em estudos clínicos e epidemiológicos. O MINI já está disponível em aproximadamente 30 idiomas, incluindo a versão brasileira. As perspectivas atuais de adaptação e aplicação transcultural do instrumento são discutidas.

Descritores Entrevistas diagnósticas padronizadas. Diagnósticos psiquiátricos. MINI.

Abstract Objectives: The MINI is a short (15-30 minutes) structured diagnostic interview compatible with DSM-III-R/ IV and ICD-10 criteria. It was designed for the clinical practice and research in psychiatric and primary care

Recebido em 5/4/2000. Aceito em 28/6/2000

Fonte de financiamento: Smithkline Beecham Pharmaceutical Company ( ${ }^{\circ}$ SB-1902), do CNAM (Caisse Nationale Assurance Maladie, França: $\left.{ }^{\circ} 701061\right)$ e do INSERM

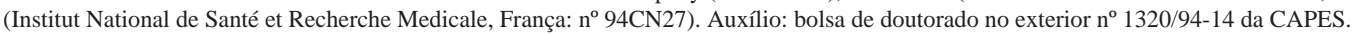

Conflito de interesse inexistente.

*Cópias e outras informações sobre a família MINI estão disponíveis na internet (www.medical-outcomes.com). Cópias da versão brasileira do MINI 5.0 (DSM-IV) podem ser obtidas com a autora. 
settings. Clinicians learn how to use it after a short training (1-3 hours). The MINI Plus is a more detailed version that helps mainly with the diagnosis of Psychotic and Mood DSM-IV Disorders. Here are presented the results of 4 MINI validation studies conducted in Europe and the U.S.

Methods: The studies 1 (France) and 2 (U.S.) tested the DSM-III-R MINI reliability (inter-rater and test-retest; $\mathrm{n}=84,42$ psychiatric patients from each center) and its validity comparing with the CIDI ( $\mathrm{n}=346,296$ psychiatric patients and 50 controls) and the SCID-P ( $=370,308$ psychiatric patients and 62 controls), respectively. The study 3 tested the validity of general practitioners' diagnoses using the MINI (DSM-IV) compared with expert psychiatrists' diagnoses for 409 patients of primary care centers in four countries (France, Spain, Italy and United Kingdom). The study 4 tested inter-rater reliability ( $n=20$ psychiatric patients) and the Psychotic Disorders, Depression and Mania modules validity of the Mini Plus - DSM-IV ( $n=104$ psychiatric patients) compared with two standard criteria: CIDI and expert psychiatrists' diagnoses. Quantitative (indexes of agreement and validity) and qualitative (sources of discrepancies) analyses were performed.

Results: The MINI (studies 1 e 2) and MINI Plus (study 4) reliability were overall satisfactory. Compared to various diagnostic standard criteria (CIDI, SCID-P, expert opinion) in different settings (psychiatric and primary care centers), MINI and the MINI Plus showed the same psychometric properties seen in more complex structured diagnostic interviews, reducing in more than 50\% the assessment time. Qualitative analyses identified diagnostic difficulties and bias related to cases, assessment methods and diagnostic criteria. Modifications were introduced to correct any identified problem and improve the psychometric properties of the MINI/MINI Plus.

Conclusions: MINI and its Plus version are adjusted to the clinical setting and for the assessment of severe cases. They represent an economic alternative for selecting patients, according to international criteria, in clinical trials and epidemiologic studies. The MINI DSM-IV 5.0 is available in almost 30 languages, including a Brazilian version. Current perspectives of the interview cross-cultural adjustments and application are discussed.

\section{Keywords Structured diagnostic interviews. Psychiatric diagnosis. MINI.}

\section{Introdução}

Os instrumentos diagnósticos padronizados mais amplamente utilizados em psiquiatria - PSE $^{1}$ (Present State Examination), $\mathrm{SCAN}^{2}$ (Schedules for Clinical Assessment in Neuropsychiatry), $\operatorname{SADS}^{3}$ (Schedule for Affective Disorders and Schizophrenia), DIS ${ }^{4}$ (Diagnostic Interview Schedule), $\mathrm{CIDI}^{5}$ (Composite International Diagnostic Interview) e $\operatorname{SCID}^{6}$ (Structured Clinical Interview for DSMIII-R) - foram elaborados para permitir a coleta precisa e exaustiva de dados clínicos e de diagnósticos no contexto da pesquisa. Entretanto, sua utilização na prática clínica é limitada pela necessidade de um treinamento extensivo dos utilizadores e pela longa duração das entrevistas (de 1h30 a 3h). Mais recentemente, alguns questionários breves também foram desenvolvidos para a triagem dos transtornos mentais em atenção primária: o PRIME-MD ${ }^{7}$ (The Primary Care Evaluation on Mental Disorders) e o SDDS-PC ${ }^{8}$ (The Symptom Driven Diagnostic System for Primary Care). O interesse pela utilização desses instrumentos em psiquiatria é todavia pequeno, já que os diagnósticos explorados se restringem aos transtornos depressivos e ansiosos, os mais freqüentes em cuidados primários.

Um questionário diagnóstico mais simples e breve que aqueles tipicamente destinados à pesquisa e mais abrangente que os instrumentos de triagem constituiria uma alternativa mais econômica para a seleção de pacientes em ensaios clínicos e poderia ser utilizado na prática clínica em psiquiatria. O MINI ${ }^{9}$ foi desenvolvido por pesquisadores do Hospital Pitié-Salpêtrière de Paris e da Universidade da Flórida para responder a esses objetivos. O MINI é um questionário breve (15-30 minutos), compatível com os critérios do DSM-III-R ${ }^{10} / \mathrm{IV}^{11}$ e da CID-10 ${ }^{12}$ (versões distintas), que pode ser utilizado por clínicos após um treinamento rápido (de $1 \mathrm{~h}$ a $3 \mathrm{~h}$ ).

Esse artigo descreve as características do MINI, apresenta os resultados de quatro estudos de validação do instrumento e discute as perspectivas atuais de sua adaptação e aplicação transcultural.

\section{Características do MINI}

O MINI é organizado por módulos diagnósticos independentes, elaborados de forma a otimizar a sensibilidade do instrumento, a despeito de um possível aumento de falso-positivos. Para permitir a redução da duração da entrevista são utilizadas as seguintes estratégias:

- a prioridade é a exploração dos transtornos atuais, de forma a guiar o clínico na escolha da terapêutica mais adaptada;

- a cotação das questões é dicotômica (SIM/NÃO);

- para todas as seções diagnósticas (exceto a seção transtornos psicóticos), uma ou duas questões de entrada que exploram critérios obrigatórios permitem excluir o diagnóstico em caso de respostas negativas;

- a disfunção induzida pelos transtornos e a exclusão de causas somáticas e/ou tóxicas dos sintomas não são sistematicamente exploradas;

- os algoritmos são integrados à estrutura do questionário, permitindo estabelecer ou excluir os diagnósticos ao longo da entrevista. 
Duas versões do MINI foram desenvolvidas para responder aos objetivos diagnósticos específicos de diferentes contextos de utilização:

1) destinado principalmente à utilização em cuidados primários e em ensaios clínicos, o MINI compreende 19 módulos que exploram 17 transtornos do eixo I do DSM-IV, o risco de suicídio e o transtorno da personalidade anti-social. Ao contrário dos outros módulos que geram diagnósticos positivos, o módulo Síndrome psicótica explora os sintomas do critério A da esquizofrenia, com o intuito de excluir a existência provável de um transtorno psicótico. A título ilustrativo, o módulo Episódio depressivo maior da versão brasileira do MINI 5.0 é apresentada no Anexo;

2) destinado à avaliação aprofundada dos transtornos mentais ao longo da vida, na clínica e na pesquisa em psiquiatria, o MINI Plus explora sistematicamente todos os critérios de inclusão e de exclusão e a cronologia (data do início e duração dos transtornos, número de episódios) de 23 categorias diagnósticas do DSM-IV. O módulo Transtornos psicóticos ${ }^{13}$ gera o diagnóstico positivo de 7 subtipos de psicoses: (1) transtorno psicótico decorrente de uma condição médica geral e/ou induzido por substâncias, (2) esquizofrenia, (3) transtorno esquizofreniforme, (4) transtorno esquizoafetivo, (5) transtorno psicótico breve, (6) transtorno delirante e (7) transtorno psicótico sem especificação.

Em estudos focados em transtornos específicos (a esquizofrenia, por exemplo), é possível utilizar o(s) módulo(s) diagnóstico(s) mais detalhado(s) correspondente(s) ao MINI Plus, juntamente com módulos mais curtos do MINI para excluir outros diagnósticos (abuso/dependência do álcool, por exemplo).

Outros instrumentos da família MINI incluem: o MINI Screen (autoquestionário para a utilização em atenção primária), o MINI Kid (versão para a avaliação diagnóstica de crianças e adolescentes) e o MINI Outcomes (MINI informatizado associado a um programa de registro do seguimento terapêutico, disponível na internet: www.medical-outcomes.com).

\section{Estudos de validação do MINI}

\section{Métodos}

Na ausência de um "gold standard" em psiquiatria, a estratégia habitual de validação de métodos de diagnóstico consiste em testar a confiabilidade entre métodos. Estabelecer a validade de procedimento (procedural validity) ${ }^{14}$ de um questionário diagnóstico significa demonstrar a existência de uma boa concordância entre os diagnósticos que ele gera e aqueles gerados por outro método diagnóstico conhecido, tomado como critério de referência. A escolha dessa referência constitui novamente um problema, já que tanto o método clínico tradicional quanto os questionários padronizados têm limitações bem documentadas, ${ }^{15-18}$ e que uma boa concordância diagnóstica pode, em parte, corresponder a erros similares dos métodos de avaliação comparados. Para amenizar os vieses das comparações de pares, após uma avaliação retrospectiva única, duas estratégias foram sugeridas para testar a validade de um novo procedimento diagnóstico: compará-lo a diferentes critérios de referência ${ }^{18}$ e compará-lo ao Best Estimate Diagnosis ${ }^{19}$ ou LEAD
Standard ${ }^{20}$ (diagnósticos consensuais de peritos, baseados na totalidade dos dados clínicos longitudinais disponíveis).

Essas sugestões foram consideradas no processo de validação do MINI e de sua versão Plus. Nos quatro estudos de validação apresentados no presente trabalho, o MINI/MINI Plus foram comparados com questionários padronizados com características distintas, amplamente utilizados em estudos internacionais - o CIDI (estudos 1 e 4) e o SCID-P (estudo 2) e com a opinião de psiquiatras (estudos 3 e 4). Além disso, foram realizadas tanto análises quantitativas quanto qualitativas, inclusive pelo procedimento do LEAD Standard (estudo 4), para permitir o estudo das fontes de erros ligados aos casos, aos métodos e aos critérios de diagnóstico estudados.

\section{Estudos 1 e 2: comparação do MINI ao CIDI e ao SCID-P}

Dois estudos paralelos foram realizados para testar a confiabilidade da versão DSM-III-R do MINI e sua validade com relação ao CIDI (França) ${ }^{21,22}$ e ao SCID-P (EUA). ${ }^{23}$

População e recrutamento. Para obter uma representação adequada dos principais transtornos mentais, o estudo visava incluir, em cada centro, 60 casos de episódio depressivo maior, 30 de episódio maníaco, 60 de transtornos ansiosos, 50 de transtornos psicóticos, 50 de abuso/dependência de álcool e/ou drogas e 50 sujeitos não apresentando transtornos psiquiátricos $a$ priori. A distribuição dos sujeitos nos grupos diagnósticos de inclusão era feita com base nos diagnósticos do CIDI (França) e do SCID (EUA). Foram recrutados pacientes com idade entre 18 e 65 anos, que se apresentaram consecutivamente em serviços psiquiátricos ou médicos (grupo controle) e que consentiram em participar do estudo. Aqueles que apresentavam demência, retardo mental ou problemas de linguagem foram excluídos.

Investigadores e treinamento. Seis investigadores (3 psiquiatras e 2 psicólogos) participaram do estudo francês, todos com experiência no uso do CIDI em estudo anterior. ${ }^{24}$ Dezesseis investigadores (10 psiquiatras, 4 assistentes de pesquisa e 2 estudantes de medicina) participaram do estudo americano. Os investigadores franceses foram treinados no uso do MINI e do CIDI e os americanos no uso do MINI e do SCID-P. Para melhorar a confiabilidade entre centros e investigadores, uma das investigadoras de Paris (E.W.) treinou os americanos no uso do CIDI e foi treinada no uso do SCID, na Flórida, e, de volta à França, treinou os franceses no uso do SCID.

Coleta de dados. O mesmo investigador aplicou o MINI seguido do CIDI (sujeitos franceses) ou do SCID-P (sujeitos americanos), durante a mesma entrevista. O CIDI e o SCID-P foram aplicados em 40 pacientes em cada centro, em ordem diferente para cada metade da amostra. Para testar a confiabilidade do MINI, 42 pacientes de cada centro foram examinados simultaneamente por dois investigadores (confiabilidade entre avaliadores) e reexaminados por um terceiro investigador 24 a 48 horas depois (confiabilidade teste-reteste).

Análise de dados. A confiabilidade do MINI foi estimada pelo coeficiente kappa. A concordância diagnóstica (kappa) e as propriedades psicométricas do MINI (sensibilidade, especificidade, valor preditivo positivo [VPP], valor preditivo nega- 
tivo [VPN] e eficiência - \% de pacientes entrevistados corretamente classificados) com relação aos questionários de referência (CIDI ou SCID-P) foram calculadas para cada um dos 17 transtornos do eixo I do DSM-III-R explorados. Para examinar em detalhe os problemas ligados ao diagnóstico das psicoses e as possibilidades de otimização dos questionários, foram realizadas análises específicas da concordância e das razões de discordância entre o MINI e o CIDI para o diagnóstico dos transtornos psicóticos e do humor. ${ }^{22}$ Nenhum estudo prévio havia analisado, sistematicamente, as causas de discordância diagnóstica entre questionários padronizados baseados na mesma criteriologia, na avaliação de pacientes psicóticos.

Estudo 3: comparação dos diagnósticos do MINI e de psiquiatras em centros de atenção primária

Centros e população. Esse estudo visava testar a concordância entre diagnósticos gerados por clínicos gerais usando o MINI (DSM-IV) e diagnósticos clínicos habituais de psiquiatras, em centros de atenção primária de quatro países (França, Espanha, Itália e Reino Unido). Aproximadamente 10 clínicos gerais em cada país concordaram em avaliar em torno de 10 pacientes. Todos os pacientes deveriam ter pelo menos 18 anos de idade. Aqueles que apresentavam demência, retardo mental ou doenças físicas graves foram excluídos.

Procedimentos. Para obter uma representação adequada dos principais transtornos mentais em serviços de atenção primária, o objetivo era selecionar um número elevado de prováveis casos. Para isso, todos os pacientes responderam o General Health Questionnaire $^{25}$ (GHQ-12) e a maioria dos que apresentavam baixos escores foram excluídos. Os pacientes selecionados para as entrevistas diagnósticas aprofundadas responderam ao MINI (11 módulos correspondendo aos transtornos mais comuns em cuidados primários), aplicado por clínicos gerais, e foram reavaliados por um psiquiatra após 1 a 3 dias. Para os propósitos desse estudo, o MINI (que foi desenvolvido simultaneamente em inglês e francês) foi traduzido (e retrotraduzido) para o espanhol e o italiano.

Diagnósticos de referência. A referência foi a opinião de peritos, todos psiquiatras conhecidos no meio científico em seus respectivos países. Os diagnósticos DSM-IV dos psiquiatras foram estabelecidos segundo os procedimentos clínicos habituais (questões abertas e múltiplas fontes de informação).

Análise de dados. A concordância diagnóstica (kappa) e as propriedades psicométricas (sensibilidade, especificidade, valores preditivos positivos [VPP] e negativos [VPN]) do MINI, aplicado por clínicos gerais, com relação aos diagnósticos clínicos dos psiquiatras (referência), foram calculadas para os diagnósticos de episódio depressivo maior, distimia, transtorno de ansiedade generalizada, transtorno de pânico com agorafobia e fobia social).

Estudo 4: comparação do MINI Plus ao CIDI e à opinião de psiquiatras para a identificação de transtornos psicóticos e do humor do DSM-IV

Esse estudo ${ }^{13,26}$ tinha por objetivos:

1) testar a validade dos módulos Transtornos psicóticos, Depressão e Mania do MINI Plus (gerando o diagnóstico de síndro- me psicótica (critério A da esquizofrenia) e de 7 subtipos de transtornos psicóticos e 5 subtipos de transtornos do humor do DSM-IV) com relação a dois critérios de referência: diagnósticos do CIDI e diagnósticos clínicos de psiquiatras;

2) examinar os problemas ligados ao diagnóstico das psicoses e as possibilidades de otimização dos procedimentos de avaliação.

População e recrutamento. Foram selecionados pacientes com idades entre 18 e 65 anos, que se apresentaram consecutivamente em serviços psiquiátricos e aceitaram participar do estudo, com exceção daqueles que apresentavam demência, retardo mental e/ou problemas de linguagem. Tendo como base o diagnóstico clínico principal dos psiquiatras, o recrutamento visou incluir:

- 50 pacientes apresentando transtornos psicóticos (transtorno psicótico decorrente de uma condição médica geral e/ou induzido por substâncias, esquizofrenia, transtorno esquizofreniforme, transtorno esquizoafetivo, transtorno psicótico breve, transtorno delirante e transtorno psicótico sem especificação);

- e 50 pacientes apresentando outros transtornos psiquiátricos (em particular transtornos do humor).

Investigadores e psiquiatras. Seis investigadores (5 psiquiatras e 1 enfermeira psiquiátrica) com boa experiência clínica e 12 psiquiatras clínicos com vários anos de exercício participaram do estudo. Todos foram treinados nos critérios do DSMIV. Os investigadores participaram de um treinamento de 5 dias no uso do MINI e do CIDI, que incluía a apresentação dos instrumentos e exercícios de cotação de vinhetas, de entrevistas em vídeo e com jogos de papéis. O treinamento nos critérios do DSM-IV e no uso dos questionários padronizados foi ministrado pela investigadora principal (P.A).

Coleta de dados. O mesmo investigador aplicou o MINI Plus (17 seções diagnósticas) e, em seguida, as seções Depressão, Mania e Psicose do CIDI, durante a mesma entrevista. No intervalo máximo de uma semana, diagnósticos clínicos independentes foram estabelecidos pelos psiquiatras, após discussão com um perito nos critérios do DSM-IV, cujo objetivo era traduzir os diagnósticos intuitivos dos psiquiatras em diagnósticos DSM-IV, sem interferir no seu raciocínio diagnóstico, para reduzir a variância de critérios de diagnóstico e melhorar a confiabilidade dos diagnósticos clínicos. Um subgrupo de 20 pacientes foi examinado simultaneamente por 2 investigadores, para testar a confiabilidade do MINI Plus.

Análise de dados. A confiabilidade do MINI Plus foi estimada pelo coeficiente kappa para a identificação de sintomas psicóticos e das seguintes categorias diagnósticas: Transtornos psicóticos, Transtornos do humor, Transtornos ansiosos, Transtornos induzidos por uso de substâncias.

No estudo da confiabilidade entre métodos, três comparações foram realizadas: MINI Plus vs CIDI (referência), MINI Plus $v s$ psiquiatras (referência) e CIDI $v s$ psiquiatras (referência). Análises quantitativas e qualitativas foram realizadas para examinar a concordância (kappa), as propriedades psicométricas (sensibilidade, especificidade, VPP, VPN e eficiência) do(s) método(s) testado(s), os níveis de 
discordância (critérios sintomatológicos, temporais, de gravidade, de exclusão) e as razões de discordância (fatores ligados ao procedimento experimental e às características dos métodos de avaliação e/ou dos critérios do DSM-IV) para os diagnósticos de transtornos psicóticos e do humor do DSM-IV (categorias gerais e específicas). Modificações foram propostas para otimizar os métodos diagnósticos e o impacto de algumas delas foi testado.

“Best Estimate Diagnosis (BED)”. O procedimento BED ${ }^{19}$ foi utilizado no intuito de aprofundar o estudo dos casos para os quais o diagnóstico principal do psiquiatra discordava dos diagnósticos gerados tanto pelo MINI Plus, quanto pelo CIDI (transtornos atuais: $n=53$; transtornos ao longo da vida: $n=46$ ). Para esses pacientes, foram elaborados prontuários resumindo todos os dados clínicos e diagnósticos disponíveis (informações provenientes do MINI Plus, do CIDI, dos psiquiatras e dados do prontuário médico recolhidos para esse fim), os quais foram analisados conjuntamente por dois psiquiatras experimentados que formularam uma conclusão diagnóstica consensual final para cada caso. Uma análise qualitativa das fontes de problemas encontrados para estabelecer o BED foi realizada, visando identificar melhor as dificuldades diagnósticas ligadas aos casos, métodos de avaliação e critérios do DSM-IV.

\section{Resultados}

Estudos 1 e 2: comparação do MINI ao CIDI e ao SCID-P

Características da população. Completaram os estudos 636 sujeitos (EUA: 330; França: 306). Como 40 sujeitos de cada centro participaram dos dois estudos, um total de 346 (296 psiquiátricos e 50 controles) foram incluídos na comparação MINI vs CIDI e 370 (308 psiquiátricos e 62 controles) foram incluídos na comparação MINI vs SCID-P. Em cada centro, os sujeitos avaliados se distribuíram igualmente por gênero. A média de idade foi de 42,2 (SD 15,1) anos para o centro francês e de 44,8 (SD 15,1) anos para o centro americano.

Duração das entrevistas. Em média, a aplicação do MINI demandou um quarto do tempo de aplicação do CIDI (21 \pm SD 7,7 minutos $v s$ 92 \pm SD 29,8 minutos) e a metade do tempo de aplicação do SCID-P $(18,7 \pm$ SD 11,6 minutos $v s$ 43 \pm SD 30,6 minutos).

Confiabilidade do MINI. A concordância entre avaliadores e teste-reteste foi $\geq 0,75$ para todos os diagnósticos, exceto para episódio maníaco atual $(0,35)$.

MINI vs CIDI. Como mostra a Tabela 1, a concordância (kappa) foi superior a 0,50 para todos os diagnósticos explorados, exceto para fobia específica e para transtorno de ansiedade generalizada. Os índices psicométricos do MINI foram satisfatórios para a maioria dos diagnósticos: sensibilidade $\geq 0,70$ (exceto para transtorno de pânico, agorafobia, fobia específica e bulimia nervosa); especificidade $\geq 0,70 ; \mathrm{VPN} \geq 0,88 ; \mathrm{VPP} \geq 0,60$ (exceto para os diagnósticos atuais de transtorno de ansiedade generalizada e fobia social e para o diagnóstico de bulimia nervosa ao longo da vida); eficiência $\geq 0,81$ (exceto para os diagnósticos atuais de transtorno de ansiedade generalizada e fobia social).

Para os transtornos psicóticos e do humor, os valores do kappa e a sensibilidade do MINI foram superiores a 0,65 , exceto para a identificação de alucinações atuais. A especificidade e os VPN foram elevados $(\geq 0,80)$ e os VPP variaram de 0,62 a 0,89 . A eficiência (proporção de classificação correta) foi elevada $(\geq 0,88)$, sobretudo devido à boa concordância para a identificação dos "não casos". Divergências em nível da data do episódio (do humor/psicótico) mais recente explicavam $40 \%$ das discordâncias para os diagnósticos atuais de episódio maníaco e síndrome psicótica (critério A da esquizofrenia) e 25\% das discordâncias para o diagnóstico de transtorno psicótico atual. Das discordâncias para episódio maníaco ao longo da vida, 53\% implicavam disparidades no que se refere à gravidade do episódio explorado: o MINI (que explora prioritariamente os episódios atuais) tendia a identificar como hipomaníacos indivíduos classificados como maníacos pelo CIDI (que explora prioritariamente o episódio mais grave ao longo da vida). A metade das discordâncias para os transtornos psicóticos ao longo da vida estava ligada às diferenças dos algoritmos dos questionários. Os erros mais freqüentes eram do CIDI, cujos algoritmos geram um número restrito de diagnósticos de transtornos psicóticos e não permitem a identificação de casos atípicos (pacientes respondendo parcialmente aos critérios para uma categoria diagnóstica específica). As modificações de algoritmos testadas melhoraram os índices de validade do MINI e do CIDI.

MINI vs SCID-P. Como mostra o Tabela 1, a concordância (kappa) foi superior a 0,50 para todos os diagnósticos explorados, exceto para a dependência de drogas atual. Os índices psicométricos do MINI foram satisfatórios: sensibilidade $\geq 0,70$ (exceto para distimia, transtorno obsessivo-compulsivo e dependência de drogas atual); especificidade e VPN $\geq 0,85$; VPP $\geq 0,60$ (exceto para os diagnósticos atuais de distimia, transtorno psicótico e dependência de drogas e para os diagnósticos atual e ao longo da vida de fobia específica e fobia social); eficiência $\geq 0,83$.

\section{Estudo 3: comparação dos diagnósticos do MINI e de psi- quiatras em centros de atenção primária}

Características da população. Completaram o estudo 409 pacientes ( \pm 100 de cada país), dos quais $62 \%$ eram mulheres e $61 \%$ preenchiam os critérios para pelo menos um dos onze diagnósticos explorados. Os transtornos mais freqüentes foram episódio depressivo maior $(39,4 \%)$, transtorno de ansiedade generalizada $(25,7 \%)$ e fobia social $(10,5 \%)$.

Concordância com os psiquiatras. Uma concordância entre os diagnósticos MINI gerados por clínicos gerais e os diagnósticos clínicos dos psiquiatras foi observada em $85 \%$ dos casos. Como mostra a Tabela 2, a concordância foi satisfatória para os transtornos mais comuns: episódio depressivo maior $(0,68)$, transtorno de ansiedade generalizada $(0,62)$ e fobia social $(0,66)$, enquanto kappas menores foram observados para distimia $(0,41)$ e transtorno de pânico com agorafobia $(0,48)$.

\section{Estudo 4: Comparação do MINI Plus ao CIDI e à opinião de psiquiatras para a identificação de Transtornos Psicóti- cos e do Humor do DSM-IV}

Características da população. 104 pacientes psiquiátricos

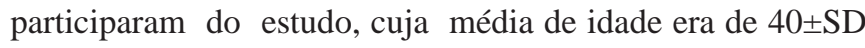


Tabela 1 - Estudos 1 e 2: comparação dos diagnósticos psiquiátricos (DSM-III-R) gerados pelo MINI àqueles gerados pelo CIDI e pelo SCID-P (critérios de referência).

\begin{tabular}{|c|c|c|c|c|c|c|c|c|}
\hline Diagnósticos & Comparação & $\begin{array}{l}\text { Casos }(n) \text { segundo } \\
\text { a referência }\end{array}$ & kappa & Sens & Espec & VPP & VPN & Efic \\
\hline \multirow[t]{2}{*}{ Episódio depressivo maior } & MINI x CIDI & $173 / 343$ & 0,73 & 0,94 & 0,79 & 0,82 & 0,93 & 0,85 \\
\hline & MINI $\times$ SCID & $169 / 370$ & 0,84 & 0,96 & 0,88 & 0,87 & 0,97 & 0,91 \\
\hline Distimia & MINI x SCID & $6 / 370$ & 0,52 & 0,67 & 0,99 & 0,45 & 0,99 & 0,98 \\
\hline \multirow{2}{*}{ Episódio maníaco atual } & $\mathrm{MINI} \times \mathrm{CIDI}$ & $21 / 342$ & 0,65 & 0,86 & 0,96 & 0,56 & 0,99 & 0,95 \\
\hline & MINI x SCID & $38 / 370$ & 0,67 & 0,82 & 0,95 & 0,63 & 0,98 & 0,93 \\
\hline \multirow{2}{*}{ Episódio maníaco ao longo da vida } & $\mathrm{MINI} \times \mathrm{CIDI}$ & $56 / 343$ & 0,63 & 0,77 & 0,92 & 0,64 & 0,95 & 0,88 \\
\hline & MINI $\times$ SCID & $74 / 370$ & 0,73 & 0,81 & 0,94 & 0,76 & 0,95 & 0,90 \\
\hline Transtorno de pânico atual & MINI x SCID & $88 / 370$ & 0,76 & 0,84 & 0,93 & 0,80 & 0,95 & 0,90 \\
\hline Transtorno de pânico ao longo da vida & MINI x SCID & $119 / 370$ & 0,80 & 0,88 & 0,93 & 0,85 & 0,94 & 0,91 \\
\hline \multirow[t]{2}{*}{ Agorafobia atual } & MINI x CIDI & $58 / 346$ & 0,58 & 0,59 & 0,95 & 0,71 & 0,92 & 0,85 \\
\hline & MINI x SCID & $87 / 370$ & 0,67 & 0,85 & 0,88 & 0,69 & 0,95 & 0,86 \\
\hline Agorafobia ao longo da vida & MINI x SCID & $109 / 370$ & 0,73 & 0,82 & 0,92 & 0,81 & 0,92 & 0,88 \\
\hline \multirow[t]{2}{*}{ Fobia social atual } & MINI x CIDI & $58 / 346$ & 0,54 & 0,72 & 0,88 & 0,55 & 0,94 & 0,72 \\
\hline & MINI $\times$ SCID & $47 / 370$ & 0,51 & 0,81 & 0,86 & 0,46 & 0,97 & 0,83 \\
\hline Fobia social ao longo da vida & MINI x SCID & $53 / 370$ & 0,60 & 0,81 & 0,90 & 0,57 & 0,97 & 0,87 \\
\hline Fobia específica atual & MINI x CIDI & $65 / 346$ & 0,43 & 0,46 & 0,93 & 0,60 & 0,88 & 0,81 \\
\hline Fobia específica ao longo da vida & MINI x SCID & $37 / 370$ & 0,55 & 0,70 & 0,93 & 0,52 & 0,97 & 0,90 \\
\hline \multirow[t]{2}{*}{ Transtorno de ansiedade generalizada } & MINI x CIDI & $48 / 345$ & 0,36 & 0,88 & 0,72 & 0,34 & 0,97 & 0,66 \\
\hline & MINI $\times$ SCID & $100 / 370$ & 0,70 & 0,91 & 0,86 & 0,71 & 0,96 & 0,86 \\
\hline Transtorno obsessivo-compulsivo & MINI x SCID & $21 / 370$ & 0,63 & 0,62 & 0,98 & 0,68 & 0,98 & 0,96 \\
\hline \multirow{2}{*}{ Dependência ao álcool atual } & MINI x CIDI & $93 / 346$ & 0,82 & 0,83 & 0,97 & 0,91 & 0,94 & 0,93 \\
\hline & MINI x SCID & $40 / 370$ & 0,67 & 0,80 & 0,95 & 0,64 & 0,98 & 0,93 \\
\hline \multirow[t]{2}{*}{ Dependência de drogas atual } & MINI x CIDI & $62 / 346$ & 0,81 & 0,89 & 0,95 & 0,81 & 0,97 & 0,94 \\
\hline & MINI x SCID & $33 / 370$ & 0,43 & 0,45 & 0,96 & 0,50 & 0,95 & 0,90 \\
\hline Dependência de drogas ao longo da vida & MINI x SCID & $62 / 370$ & 0,64 & 0,77 & 0,92 & 0,65 & 0,95 & 0,88 \\
\hline \multirow{3}{*}{$\begin{array}{l}\text { Anorexia nervosa } \\
\text { Bulimia nervosa ao longo da vida }\end{array}$} & MINI x SCID & $10 / 370$ & 0,90 & 0,90 & 1,00 & 0,90 & 1,00 & 0,99 \\
\hline & MINI x CIDI & $24 / 346$ & 0,53 & 0,63 & 0,96 & 0,52 & 0,97 & 0,93 \\
\hline & MINI $\times$ SCID & $12 / 370$ & 0,78 & 0,92 & 0,99 & 0,69 & 1,00 & 0,98 \\
\hline Transtorno de estresse pós-traumático & MINI x SCID & $59 / 370$ & 0,78 & 0,85 & 0,96 & 0,82 & 0,97 & 0,93 \\
\hline \multirow[t]{2}{*}{ Transtorno psicótico atual } & $\mathrm{MINI} \times \mathrm{CIDI}^{*}$ & $44 / 303$ & 0,72 & 0,89 & 0,93 & 0,67 & 0,98 & 0,91 \\
\hline & MINI x SCID & $37 / 370$ & 0,53 & 0,84 & 0,89 & 0,46 & 0,98 & 0,88 \\
\hline \multirow[t]{2}{*}{ Transtorno psicótico ao longo da vida } & $\mathrm{MINI} \times \mathrm{CIDI}^{*}$ & $67 / 303$ & 0,71 & 0,87 & 0,90 & 0,72 & 0,96 & 0,88 \\
\hline & MINI x SCID & $82 / 370$ & 0,76 & 0,88 & 0,92 & 0,77 & 0,96 & 0,91 \\
\hline Síndrome psicótica atual & $\mathrm{MINI} \times \mathrm{CIDI}^{*}$ & $41 / 305$ & 0,68 & 0,90 & 0,91 & 0,62 & 0,98 & 0,90 \\
\hline Alucinações & & $30 / 305$ & 0,44 & 0,37 & 0,98 & 0,73 & 0,93 & 0,92 \\
\hline Sintomas psicóticos ao longo da vida & $\mathrm{MINI} \times \mathrm{CIDI}^{*}$ & $84 / 306$ & 0,82 & 0,87 & 0,95 & 0,87 & 0,95 & 0,92 \\
\hline Delírios & & 76 / 306 & 0,82 & 0,84 & 0,96 & 0,89 & 0,95 & 0,93 \\
\hline Alucinações & & $54 / 306$ & 0,72 & 0,68 & 0,98 & 0,86 & 0,93 & 0,92 \\
\hline
\end{tabular}

SENS = sensibilidade; ESPEC = especificidade; VPP = valor preditivo positivo; VPN = valor preditivo negativo; EFIC = eficiência

*Dados apenas da amostra francesa

Tabela 2 - Estudo 3: comparação dos diagnósticos psiquiátricos gerados por clínicos gerais usando o MINI (DSM-IV), aos diagnósticos clínicos de psiquiatras (critério de referência), em centros de atenção primária $(n=409)$.

\begin{tabular}{lrrrrr}
\hline \multicolumn{1}{c}{ kappa } & \multicolumn{1}{c}{ Sens } & \multicolumn{1}{c}{ Espec } & VPP & VPN \\
\hline $\begin{array}{l}\text { Episódio depressivo maior } \\
\text { Distimia }\end{array}$ & 0,68 & 0,86 & 0,84 & 0,75 & 0,92 \\
$\begin{array}{l}\text { Transtorno de ansiedade } \\
\text { Generalizada }\end{array}$ & 0,41 & 0,41 & 0,96 & 0,54 & 0,93 \\
Transtorno de pânico com & 0,62 & 0,67 & 0,92 & 0,79 & 0,97 \\
agorafobia & 0,48 & 0,44 & 0,97 & 0,70 & 0,90 \\
Fobia social & 0,66 & 0,83 & 0,95 & 0,58 & 0,99
\end{tabular}

SENS = sensibilidade; ESPEC = especificidade; VPP = valor preditivo positivo;

VPN $=$ valor preditivo negativo

$\mathrm{z} 11,4$ anos e dos quais $49 \%$ eram mulheres, $51 \%$ apresentavam transtorno psicótico (diagnóstico de inclusão dos psiquiatras) e $61 \%$ foram recrutados em unidades de hospitalização (enfermarias ou hospital-dia).

Duração das entrevistas. A média de duração da aplicação completa do MINI Plus (17 seções) foi de 29+SD 9,8 minutos

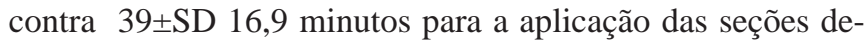
pressão, mania e psicose do CIDI.
Confiabilidade do MINI Plus. A concordância entre avaliadores foi satisfatória tanto para as categorias diagnósticas $(0,86$ a 1) quanto para os sintomas psicóticos $(0,62$ a 0,95$)$ examinados, exceto para o comportamento desorganizado $(0,31)$.

MINI Plus vs CIDI (referência). Como mostra a Tabela 3 a concordância foi superior a 0,51 para os diagnósticos avaliados, exceto para os transtornos psicóticos atuais $(0,49)$ e a esquizofrenia atual $(0,45)$.

Os índices psicométricos do MINI Plus foram comparáveis aos de outros questionários padronizados mais longos: sensibilidade $\geq 0,64$ (exceto para o transtorno depressivo maior atual); especificidade $\geq 0,71 ; \mathrm{VPN} \geq 0,84$; VPP $\geq 0,60$, exceto para os diagnósticos atuais de esquizofrenia $(0,44)$, transtornos psicóticos $(0,44)$ e transtornos do humor $(0,53)$; eficiência $\geq$ 0,76 . As discordâncias situavam-se principalmente no que se refere à avaliação da cronologia dos transtornos - data do episódio (psicótico/do humor) mais recente (transtornos atuais) e diagnóstico diferencial entre transtornos psicóticos e do humor (transtornos ao longo da vida) - e dos algoritmos para os transtornos psicóticos. Tendo em conta as fontes de erro iden- 
tificadas, as seguintes proposições objetivas foram feitas para melhorar a validade dos questionários:

MINI Plus: (a) aumento do peso do julgamento clínico do entrevistador para a avaliação da sintomatologia psicótica e dos critérios de exclusão; (b) exploração dos episódios psicóticos antes dos episódios do humor; (c) exploração da cronologia dos transtornos psicóticos e do humor com relação a eventos de vida marcantes; (d) consideração da existência e duração de um tratamento antipsicótico para estabelecer a gravidade e a duração total da psicose (critérios B e C da esquizofrenia); (e) introdução da noção de diagnóstico provisório, em caso de dúvida.

Algoritmos do CIDI: (a) em presença de um transtorno psicótico ao longo da vida, consideração da existência de sintomas psicóticos observados durante a entrevista para estabelecer o diagnóstico de transtorno psicótico atual; (b) introdução da categoria de transtorno psicótico sem especificação para permitir a identificação de pacientes psicóticos que preenchem parcialmente os critérios de diagnóstico para esquizofrenia, transtorno esquizofreniforme e transtorno esquizoafetivo.

MINI Plus e CIDI vs diagnósticos clínicos dos psiquiatras (referência). Como mostra a Tabela 3, a concordância foi modesta (MINI Plus: 0,30 a 0,61; CIDI: 0,20 a 0,48), mas comparável àquela observada em estudos similares. Os questionários identificaram menos transtornos psicóticos e mais transtornos do humor que os psiquiatras. O MINI Plus mostrou-se mais sensível que o CIDI para identificar os transtornos atuais e os transtornos psicóticos diagnosticados pelos psiquiatras, a especificidade dos dois questionários sendo satisfatória. A proporção de classificação correta (eficiência) variou de 0,68 a
0,88 para o MINI e de 0,65 a 0,86 para o CIDI.

Para os transtornos psicóticos, as discordâncias decorriam sobretudo de vieses das informações fornecidas pelos pacientes, envolvendo múltiplos critérios ao mesmo tempo: tendência a negar ou minimizar a psicose ou ainda a apresentá-la como um estado depressivo. Para os transtornos do humor, as razões de discordância eram heterogêneas: os erros dos questionários no que se refere ao diagnóstico diferencial entre transtornos psicóticos e do humor eram freqüentes; os psiquiatras subexploravam os sintomas afetivos e, diferentemente dos questionários, utilizavam uma estratégia diagnóstica hierárquica. A sensibilidade das versões modificadas do MINI Plus e do CIDI para o diagnóstico de esquizofrenia e de transtornos psicóticos (categoria ampla) foi $10 \%$ a $30 \%$ mais elevada que a sensibilidade das versões originais dos questionários, confirmando o interesse das modificações propostas. Também a utilização de questionários padronizados breves, de forma complementar aos procedimentos clínicos habituais, poderia reduzir os erros diagnósticos dos psiquiatras decorrentes da exploração incompleta da sintomatologia.

"Best Estimate Diagnosis". Foi difícil ou impossível estabelecer o BED para aproximadamente dois terços dos casos discordantes de esquizofrenia, transtorno depressivo maior e transtorno bipolar, os quais implicavam mais freqüentemente divergências em relação ao diagnóstico diferencial entre categorias ou subcategorias dos transtornos. As dificuldades encontradas para estabelecer o BED estavam ligadas à complexidade dos casos, à insuficiência dos dados disponíveis provenientes dos questionários e das fontes clínicas (psiquiatras e prontuário médico), mas também aos problemas práticos de aplicação das de-

Tabela 3 - Estudo 4: comparação dos diagnósticos de síndrome psicótica, transtornos psicóticos e do humor do DSM-IV gerados pelo MINI Plus (MP), pelo CIDI e por psiquiatras (PQ).

\begin{tabular}{|c|c|c|c|c|c|c|c|c|}
\hline Diagnósticos & $\begin{array}{l}\text { Comparação } \\
\text { a referência }(*)\end{array}$ & Casos (n) segundo & kappa & Sens & Espec & VPP & VPN & Efic \\
\hline \multirow{3}{*}{$\begin{array}{l}\text { Síndrome psicótica } \\
\text { ao longo da vida }\end{array}$} & $\mathrm{MP} \times \mathrm{CIDI} *$ & $57 / 104$ & 0,88 & 0,95 & 0,94 & 0,95 & 0,94 & 0,94 \\
\hline & $M P \times P Q *$ & $50 / 95$ & 0,30 & 0,32 & 0,96 & 0,87 & 0,65 & 0,85 \\
\hline & CIDI x PQ* & $50 / 95$ & 0,23 & 0,23 & 0,98 & 0,91 & 0,62 & 0,80 \\
\hline \multirow{3}{*}{$\begin{array}{l}\text { Esquizofrenia } \\
\text { atual }\end{array}$} & MP $\times$ CIDI* & $11 / 101$ & 0,45 & 0,64 & 0,90 & 0,44 & 0,95 & 0,87 \\
\hline & $M P \times P Q *$ & $44 / 101$ & 0,30 & 0,32 & 0,96 & 0,87 & 0,65 & 0,68 \\
\hline & CIDI x PQ* & $44 / 101$ & 0,23 & 0,23 & 0,98 & 0,91 & 0,62 & 0,65 \\
\hline \multirow{3}{*}{$\begin{array}{l}\text { Esquizofrenia } \\
\text { ao longo da vida }\end{array}$} & $\mathrm{MP} \times \mathrm{CIDI} *$ & $55 / 103$ & 0,64 & 0,68 & 0,93 & 0,77 & 0,90 & 0,86 \\
\hline & $M P \times P Q *$ & $45 / 103$ & 0,35 & 0,40 & 0,93 & 0,82 & 0,67 & 0,70 \\
\hline & CIDI x PQ* & $45 / 103$ & 0,42 & 0,47 & 0,93 & 0,84 & 0,69 & 0,73 \\
\hline \multirow{3}{*}{$\begin{array}{l}\text { Transtorno psicótico } \\
\text { atual }\end{array}$} & $\mathrm{MP} \times \mathrm{CIDI} *$ & $14 / 102$ & 0,49 & 0,86 & 0,83 & 0,44 & 0,97 & 0,83 \\
\hline & $M P \times P Q *$ & $50 / 102$ & 0,39 & 0,46 & 0,92 & 0,85 & 0,64 & 0,70 \\
\hline & CIDI $x$ PQ* & $50 / 102$ & 0,20 & 0,24 & 0,96 & 0,86 & 0,57 & 0,61 \\
\hline \multirow{3}{*}{$\begin{array}{l}\text { Transtorno psicótico } \\
\text { ao longo da vida }\end{array}$} & $\mathrm{MP} \times \mathrm{CIDI} *$ & $35 / 104$ & 0,51 & 0,83 & 0,72 & 0,60 & 0,89 & 0,76 \\
\hline & $M P \times P Q *$ & $54 / 104$ & 0,43 & 0,67 & 0,76 & 0,75 & 0,69 & 0,71 \\
\hline & CIDI x PQ* & 54 / 104 & 0,45 & 0,55 & 0,90 & 0,86 & 0,65 & 0,72 \\
\hline \multirow{2}{*}{$\begin{array}{l}\text { Transtorno depressivo maior } \\
\text { atual }\end{array}$} & $\mathrm{MP} \times \mathrm{CIDI} *$ & $19 / 104$ & 0,58 & 0,58 & 0,95 & 0,73 & 0,91 & 0,88 \\
\hline & $M P \times P Q *$ & $17 / 104$ & 0,56 & 0,59 & 0,94 & 0,67 & 0,92 & 0,88 \\
\hline \multirow{3}{*}{$\begin{array}{l}\text { Transtorno depressivo maior } \\
\text { ao longo da vida }\end{array}$} & $\mathrm{MP} \times \mathrm{CIDI} *$ & $34 / 104$ & 0,52 & 0,68 & 0,84 & 0,68 & 0,84 & 0,79 \\
\hline & $M P \times P Q *$ & 27 / 104 & 0,42 & 0,67 & 0,79 & 0,53 & 0,87 & 0,76 \\
\hline & CIDI x PQ* & $27 / 104$ & 0,47 & 0,70 & 0,80 & 0,56 & 0,89 & 0,78 \\
\hline \multirow{3}{*}{$\begin{array}{l}\text { Transtorno bipolar } \\
\text { ao longo da vida }\end{array}$} & $\mathrm{MP} \times \mathrm{CIDI} *$ & $13 / 99$ & 0,67 & 0,92 & 0,91 & 0,60 & 0,99 & 0,91 \\
\hline & $M P \times P Q *$ & $9 / 99$ & 0,33 & 0,67 & 0,84 & 0,30 & 0,96 & 0,83 \\
\hline & CIDI $\times$ PQ* & $9 / 99$ & 0,29 & 0,44 & 0,90 & 0,31 & 0,94 & 0,86 \\
\hline \multirow{3}{*}{$\begin{array}{l}\text { Transtorno do humor } \\
\text { atual }\end{array}$} & $\mathrm{MP} \times \mathrm{CIDI} *$ & $24 / 101$ & 0,51 & 0,75 & 0,82 & 0,53 & 0,91 & 0,80 \\
\hline & $M P \times P Q *$ & $31 / 101$ & 0,61 & 0,74 & 0,87 & 0,72 & 0,88 & 0,83 \\
\hline & CIDI $x$ PQ* & $31 / 101$ & 0,48 & 0,55 & 0,90 & 0,71 & 0,82 & 0,79 \\
\hline \multirow{3}{*}{$\begin{array}{l}\text { Transtorno do humor } \\
\text { ao longo da vida }\end{array}$} & $\mathrm{MP} \times \mathrm{CIDI} *$ & $50 / 102$ & 0,61 & 0,90 & 0,71 & 0,75 & 0,88 & 0,80 \\
\hline & $M P \times P Q *$ & 44 / 102 & 0,41 & 0,84 & 0,59 & 0,61 & 0,83 & 0,70 \\
\hline & CIDI x PQ* & 44 / 102 & 0,45 & 0,75 & 0,71 & 0,66 & 0,79 & 0,72 \\
\hline
\end{tabular}

$\overline{\mathrm{SENS}}=$ sensibilidade; $\mathrm{ESPEC}=$ especificidade; $\mathrm{VPP}=$ valor preditivo positivo; VPN = valor preditivo negativo; EFIC = eficiência 
finições categoriais do DSM-IV em vários casos.

Esse estudo evidencia:

(a) a necessidade de considerar informações longitudinais provenientes de múltiplas fontes para melhorar a validade da avaliação diagnóstica dos transtornos psicóticos e do humor;

(b) os limites da validade discriminante dos critérios do DSMIV, em particular para separar esquizofrenia com sintomas negativos proeminentes e transtorno depressivo maior com aspectos psicóticos; transtornos esquizoafetivo e bipolar; transtornos do Humor e da personalidade.

\section{Conclusão}

Informações mais detalhadas sobre os estudos de validação são apresentadas em outros artigos. ${ }^{9,21-23,26}$ Artigos referentes ao estudo 4 estão em curso de publicação.

Considerando os dados da literatura, os resultados referentes à confiabilidade e à validade do MINI são globalmente satisfatórios. O MINI apresenta qualidades psicométricas comparáveis às de outros questionários diagnósticos padronizados mais longos e complexos, permitindo a avaliação rápida (15-30 minutos) dos principais transtornos do eixo I do DSM-III/IV. Comparado ao CIDI e ao SCID-P, o MINI mostrou-se globalmente hiperinclusivo para gerar diagnósticos. Entretanto, a análise das razões de discordância mostrou que parte dos resultados falso-positivos (sobretudo para os transtornos psicóticos) decorria, de fato, da reduzida sensibilidade do instrumento de referência (sobretudo o CIDI) ou ainda de diferenças entre os questionários no que se refere às regras diagnósticas de inclusão (p.ex., divergências quanto à duração total das perturbações exigida para o diagnóstico do transtorno de ansiedade generalizada e da dependência de substâncias) ou de exclusão (ao contrário do MINI, o CIDI e o SCID-P excluem o diagnóstico de fobia social e fobia específica para pacientes apresentando transtorno psicótico). Para uma redução de $50 \%$ ou mais no tempo de avaliação, com relação aos questionários longos, a sensibilidade e a especificidade do MINI foram bastante satisfatórias.

O estudo 4 evidenciou, em consonância com a literatura, a reduzida sensibilidade dos questionários padronizados para o diagnóstico dos transtornos psicóticos. Dentro desses limites, o MINI Plus se mostrou mais sensível que o CIDI para identificar os casos (sobretudo atuais) de psicose diagnosticados pelos psiquiatras, sem prejuízo da especificidade. Esse melhor desempenho do MINI Plus está intimamente ligada aos objetivos e características do instrumento: brevidade, simplicidade, prioridade dada à exploração de transtornos atuais e algoritmos abrangentes, permitindo a identificação de casos típicos e atípicos de transtornos psicóticos. Além disso, algumas das modificações já integradas ao instrumento favorecem a sensibilidade e a validade do instrumento. O MINI Plus é o único questionário padronizado de aplicação rápida atualmente disponível para a avaliação dos transtornos psicóticos. Ele é assim melhor adaptado ao contexto clínico e à avaliação de pacientes mais graves, e representa uma alternativa econômica para a seleção de pacientes, segundo critérios internacionais, em estudos clínicos e epidemiológicos.

Os estudos descritos apresentaram limitações. O número reduzido de "casos" para alguns diagnósticos pode ter contribuído para o aumento dos falso-positivos. Por outro lado, a apli- cação do MINI e dos questionários diagnósticos tomados como referência - CIDI (estudos 1 e 4) e SCID-P (estudo 2) - pelo mesmo investigador e no curso da mesma entrevista, pode ter favorecido a concordância diagnóstica.

\section{Progressos e perspectivas de desenvolvimento do MINI}

Com base nos resultados dos estudos de validação, modificações foram introduzidas para corrigir as insuficiências identificadas e otimizar as propriedades psicométricas do MINI e do MINI Plus.

O MINI foi o primeiro questionário diagnóstico padronizado a ser atualizado para a exploração dos novos critérios do DSM-IV. Uma versão DSM-IV informatizada do MINI já está disponível (www.medical-outcomes.com). A versão do MINI, compatível com os critérios da CID-10, existe atualmente em inglês e francês e a elaboração da versão brasileira está em curso pela autora deste trabalho.

O eixo atual do desenvolvimento do MINI é sua adaptação e aplicação transcultural. Um projeto de colaboração internacional para a tradução do MINI Screen e das versões DSM-IV do MINI e do MINI Plus em 30 idiomas está em fase de conclusão. Todas as versões do instrumento estarão disponíveis gratuitamente na internet (www.medical-outcomes.com). Atualmente, o MINI está sendo amplamente utilizado para a seleção de pacientes em ensaios psicofarmacológicos multicêntricos e outros protocolos clínicos, em psiquiatria e em atenção primária, na Europa, nos EUA e também no Brasil.

Os trabalhos de adaptação (tradução e validação) e aplicação do instrumento em países de língua portuguesa são coordenados pela autora do presente estudo. A versão portuguesa, traduzida e retrotraduzida para o francês por um grupo de psiquiatras bilíngües, é atualmente objeto de um estudo multicêntrico (Lisboa, Porto, Coimbra e Faro) de validação com relação à opinião de psiquiatras, em centros de atenção primária. Para o desenvolvimento da versão brasileira do MINI, a metodologia combina procedimentos epidemiológicos e antropológicos, de forma a otimizar a sensibilidade cultural do instrumento. Uma versão DSM-IV preliminar (4.4) do MINI foi traduzida para o português e sua aplicabilidade (reações dos pacientes ao questionário, identificação de problemas técnicos e/ ou de compreensão) foi testada num estudo envolvendo 32 pacientes psiquiátricos em São Paulo, Goiânia e Salvador. Vinte desses pacientes, apresentando transtornos psicóticos e/ou do humor, passaram ainda por uma entrevista livre (método etnográfico) que buscou identificar seus modelos de compreensão da doença, de forma a considerá-los na formulação das questões e instruções de aplicação da versão brasileira do MINI. Os resultados desses dois estudos estão em curso de análise. A tradução brasileira da versão mais atual (5.0) do MINI DSMIV já foi realizada pela autora do presente trabalho.

As próximas etapas de adaptação do MINI ao contexto brasileiro incluem: (a) retrotradução da versão do MINI DSMIV $(5,0)$ e tradução/retrotradução do MINI Plus DSM-IV $(5,0)$ e do MINI CIM-10 por profissionais de saúde mental e antropólogos, tendo em conta as informações dos estudos acima citados; (b) implementação de estudos multicêntricos de va- 
lidação da versão brasileira do MINI em psiquiatria e em centros de atenção primária. Procedimentos para a viabilização técnica e o financiamento desse projeto estão em curso.

\section{Aplicações potenciais do MINI}

Pesquisa: o MINI pode ser utilizado para a seleção rápida de populações homogêneas em ensaios clínicos e estudos epidemiológicos ou ainda para a avaliação breve de critérios diagnósticos em estudos longitudinais.

Prática clínica: o MINI foi concebido como um "exame complementar" para permitir a coleta sistemática de informações necessárias ao estabelecimento ou à confirmação de hipóteses diagnósticas, segundo critérios precisos. O instrumento pode ser utilizado para melhorar a precisão do diagnóstico e a adequação

\section{Referências}

1. Wing JK, Cooper JE, Sartorius N. The Measurement and Classification of Psychiatric Symptoms: An Instructional Manual for the PSE and Catego Program. New York/ Cambridge: University Press; 1974.

2. World Health Organisation [WHO]. Schedules for Clinical Assessment in Neuropsychiatry (SCAN). Geneva: WHO; 1992.

3. Endicott J, Sptizer RL. A Diagnostic Interview: the Schedule for Affective Disorders and Schizophrenia. Arch Gen Psychiatry 1978;35:837-44.

4. Robins LN, Helzer JE, Croughan J, Ratcliff KS. National Institut of Mental Health Diagnostic Interview Schedule: Its History, Characteristics and Validity. Arch Gen Psychiatry 1981;38:381-9.

5. World Health Organisation [WHO]. The Composite International Diagnostic Interview (CIDI). Authorized Core Version 1.0. Geneva:WHO; 1990.

6. Spitzer RL, Williams JBW, Gibbon M, First MB. Structured Clinical Interview for DSM-III-R - Patient Version (SCID-P, 4/1/88). New York: Biometrics Research Department, New York State Psychiatric Institute; 1988.

7. Spitzer RL, Williams JBW, Kroenke K. The PRIME-MD 1000 study: validation of a new system for diagnosing mental disorders in primary care. McLean VA. Present at the Seventh Annual NIMH International Research Conference on Mental Health Problems in the General Health Section; september 21; 1993.

8. Broadhead WE, Leon AC, Weissman MM, Barret JE, Blacklow RS, Gilbert TT et al. Development and validation of the SDDS-PC screen for multiple mental disorders in primary care. Arch Fam Med 1995;4:211-9.

9. Sheehan D, Lecrubier Y, Sheehan KH, Amorim P, Janavs J, Weiller E et al. The Mini International Neuropsychiatric Interview (MINI): The Development and Validation of a Structured Diagnostic Psychiatric Interview for DSM-IV and ICD-10. J Clin Psychiatry 1998;59(suppl 20);22-33.

10. American Psychiatric Association [APA]. Diagnostic and Statistical Manual of Mental Disorders. 3rd ed (DSM-III-R). Washington (DC): APA; 1987.

11. American Psychiatric Association [APA]. Diagnostic and Statistical Manual of Mental Disorders. 4th ed (DSM-IV). Washington (DC): APA; 1994.

12. World Health Organisation [WHO]. The International Classification of Diseases and Related Health Problems.10th Revision. Geneva: WHO; 1992.

13. Amorim P. Le Processus Diagnostique des Troubles Psychotiques. Approche Standardisée et Approche Clinique [dissertation]. Paris (France): Université de Paris VI; 1998.

14. Spitzer RL, Williams JBW. Classification of mental disorders. In: Comprehensive Textbook of Psychiatry/IV 4th ed. Kaplan \& Sadoch, eds. Baltimore/London: Williams \& Wilkins Company; 1985. vol 1. p. 591-613.

15. Brockington IF, Meltzer HY. Documenting an episode of psychiatric illness: need for multiple information sources, multiple raters, and narrative. Schizophr Bull 1982;8(3):485-92.

16. Rogler LH, Malgady RG, Tryon WW. Evaluation of mental health issues of memory in the diagnostic interview schedule. J Nerv Ment Dis 1992;180:215-22. da escolha terapêutica em atenção primária e em psiquiatria.

Gestão de programas de saúde: o MINI pode ser utilizado para gerar uma base de dados clínicos e diagnósticos útil para o planejamento e a otimização de programas de atenção em saúde mental.

Ensino: no contexto da formação, o MINI pode ser utilizado para capacitar estudantes e profissionais de saúde na utilização das novas classificações internacionais e técnicas de avaliação diagnóstica dos transtornos mentais.

\section{Agradecimentos}

A Miguel Roberto Jorge (Unifesp/EPM), pela leitura crítica da versão preliminar desse trabalho e pelas valiosas sugestões para aprimorá-lo.

17. Fenning S, Bromet EJ, Jandorf L, Schwartz JE, Lavelle J, Ram R. Eliciting Psychotic Symptoms Using a Semi-Structured Diagnostic Interview. The Importance of Collateral Sources of Information in a First-Administration Sample. J Nerv Ment Dis 1994;181:20-6.

18. Wittchen HU, Semler G, Von Zerssen D. A Comparison of two diagnostic methods: clinical ICD vs DSM-III and research diagnostic criteria using the diagnostic interview schedule (Version 2). Arch Gen Psychiatry 1985;42:677-84.

19. Leckman JF, Sholomskas D, Thompson WD, Belanger A, Weissman MM. Best estimate of lifetime psychiatric diagnosis. Arch Gen Psychiatry 1982;39:879-83.

20. Sptizer RL. Psychiatric diagnosis: are clinicians still necessary? Compr Psychiatry 1983;24(5):399-411.

21. Lecrubier Y, Sheehan D, Weiller E, Amorim P, Bonora I, Sheehan K et al. The Mini international neuropsychiatric interview (MINI). A short diagnostic structured interview: reliability and validity according to the CIDI. Eur Psychiatry 1997;12:224-31.

22. Amorim P, Lecrubier Y, Weiller E, Hergueta T, Sheehan D. DSM-III-R psychotic disorders: procedural validity of the mini international neuropsychiatric interview (MINI). Concordance and causes for discordances with the CIDI. Eur Psychiatry 1998;13:26-34.

23. Sheehan D, Lecrubier Y, Sheehan KH, Janavs J, Weiller E, Keskiner A et al. The validity of the mini international neuropsychiatric interview (MINI) according to the SCID-P and its reliability. Eur Psychiatry 1997;12:232-41.

24. Sartorius N, Üstün TB, Korten A, Cooper JE, Van Drimmelen J. Progress toward achieving a common language in psychiatry, II: Results from the International field trial of the ICD-10 Diagnostic Criteria for Mental and Behavioral Disorders. Am J Psychiatry 1995;152(10):1427-37.

25. Goldberg D, Williams P. A Users Guide to the General Health Questionnaire: GHQ. Windsor: NFER-NELSON; 1988.

26. Amorim P. Critérios do DSM-IV: problemas e possibilidades de melhora da validade do diagnóstico de esquizofrenia gerado por métodos padronizados e clínicos de avaliação. In: Associação Brasileira de Psiquiatria, editors. Cidadania e direito à saúde mental. Coletânia Symposium, Série Medicina \& Saúde. São Paulo: Frôntis Editorial; 1998. p. 123-34.

\section{Correspondência}

Patrícia Amorim

Rua R-11 n³71 apto. 601

74120-030 Goiânia, GO

Tel.: (0xx62) 524-1802

Fax: (0xx62) 2854360

E-mail: p.amorim@persogo.com.br 
Anexo: Módulo Episódio Depressivo Maior da versão brasileira do MINI 5.0. (DSM-IV)*

レ: IR DIRETAMENTE aO(s) QUADRO(s) DIAGNÓSTICO(s), ASSINALAR Não EM CADA UM E PASSAR AO MÓDULO SEGUINTE

\section{A. EPISÓDIO DEPRESSIVO MAIOR}

A1 Nas duas últimas semanas, sentiu-se triste, desanimado(a), deprimido(a), durante a maior parte do dia, quase todos os dias?

A2 Nas duas últimas semanas, teve, quase todo tempo, o sentimento de não ter mais gosto por nada, de ter perdido o interesse e o prazer pelas coisas que lhe agradam habitualmente?

\section{A1 OU A2 SÃO COTADAS SIM ?}

NÃO SIM 2

NÃO SIM

\section{A3 Durante as duas últimas semanas, quando se sentia deprimido(a) / sem interesse pela} maioria das coisas:

a O seu apetite mudou de forma significativa, ou o seu peso aumentou ou diminuiu sem que o tenha desejado ? (variação de $\pm 5 \%$ ao longo do mês, isto é, $\pm 3,5 \mathrm{Kg}$, para uma pessoa de $65 \mathrm{Kg}$ ) COTAR SIM, SE RESPOSTA SIM num CASO OU No Outro

NÃO SIM 3

b Teve problemas de sono quase todas as noites (dificuldade em pegar no sono, acordar no meio da noite ou muito cedo, dormir demais)?

NÃO SIM 4

c Falou ou movimentou-se mais lentamente que de costume ou pelo contrário, sentiu-se agitado(a) e incapaz de ficar sentado quieto, quase todos os dias?

d Sentiu-se a maior parte do tempo cansado(a), sem energia, quase todos os dias?

e Sentiu-se sem valor ou culpado(a), quase todos os dias?

f Teve dificuldade para concentrar-se ou tomar decisões, quase todos os dias?

g Teve, por várias vezes, pensamentos ruins como, por exemplo, pensar que seria melhor estar morto(a) ou pensar em fazer mal a si mesmo(a)?

\begin{tabular}{lll} 
NÃO & SIM & 5 \\
NÃO & SIM & 6 \\
NÃO & SIM & 7 \\
NÃO & SIM & 8 \\
NÃO & SIM & 9 \\
\hline $\begin{array}{c}\text { NÃO } \\
\text { SIM } \\
\text { EPISÓDIO DEPRESSIVO } \\
\text { MAIOR ATUAL }\end{array}$ \\
\hline
\end{tabular}

Se o(a) ENTREVistado(A) apresenta um Episódio Depressivo Maior Atual :

A5a Ao longo da sua vida, teve outros períodos de 2 semanas ou mais, em que se sentiu deprimido (a) ou sem interesse pela maioria das coisas e durante os quais teve os problemas dos quais falamos [ SINTOMAS EXPLORADOS DE A3a à A3g ]?

$\begin{array}{llr}\begin{array}{lll}\downarrow \\ \text { NÃO }\end{array} & \text { SIM } & 10 \\ \text { NÃO } & \text { SIM } & 11 \\ \text { NÃO } & \text { SIM } \\ \begin{array}{c}\text { EPISÓDIO DEPRESSIVO } \\ \text { MAIOR PASSADO }\end{array}\end{array}$

* (C) 1992, 1994, 1998 Sheehan, Lecrubier et al. Tradução: P. Amorim 